\title{
Oral Anticoagulants in Orthopaedic Surgeries Comparison of Rivaroxaban and Standard-of-Care
}

\author{
Dr. Sanjay Khot, Dr. Sunil Kant Kamra* \\ Assistant Professor Department of Anaesthesiology Chandulal Chandrakar Memorial Medical College, \\ Kachandur, Durg (Chhattisgarh) 490024 \\ Address for Correspondence \\ Dr. Sunil Kant Kamra \\ Assistant Professor Dept. of Anaesthesiology \\ Chandulal Chandrakar Memorial Medical College, Kachandur, Durg (Chhattisgarh) 490024
}

\begin{abstract}
$\underline{\text { Abstract }}$
Background: Venous thromboembolic (VTE), deep vein thrombosis (DVT) and pulmonary embolism (PE), cause significant morbidity, mortality and healthcare costs. Direct oral anticoagulant which has been demonstrated in clinical trials, anaesthetists need to be careful of how to minimize the risks of bleeding complications when treating patients who are taking an anticoagulant. Rivaroxaban, non-vitamin K antagonist direct oral anticoagulants (DOACs), was shown to be more effective regimens for the prevention of VTE after orthopaedic surgery. Aims and objectives: To look for the influence of timing of the first thromboprophylactic dose and clinical outcomes in patients undergoing orthopaedic surgery Method: Patients aged $\geq 18$ years, with planned orthopaedic surgery or fracture-related orthopaedic surgery and in whom thromboprophylaxis has been indicated. Out of 324 patients selected for the study 164 received rivaroxaban 10 mg once daily and 160 received standard-of-care (SOC) pharmacological prophylaxis. Incidences of symptomatic thromboembolic events and bleeding events were analysed. Bleeding events and thromboembolic events recorded and calculated for the rivaroxaban and SOC groups. Results: Overall major bleeding events observed in rivaroxaban group was 9 (5.49\%) was much lower than the SOC group which was 13 (8.13\%).The percentage of patients using mechanical methods alongside pharmacological thromboprophylaxis was slightly higher in the rivaroxaban as compared to SOC groups ( 64\% and 55\%). Overall thromboembolic effect was lower in rivaroxaban as compared to SOC group. Conclusion: Comparison of rivaroxaban \& SOC shows the effectiveness and safety of rivaroxaban in patients undergoing major orthopedic surgery in clinical practice. Major bleeding events \& thromboembolic with rivaroxaban were less as compared to SOC group.
\end{abstract}

\section{Introduction}

The use of direct oral anticoagulants which includes apixaban, rivaroxaban, and dabigatran, are approved for several therapeutic indications, can simplify perioperative and postoperative management of anticoagulation. American Society of Regional Anesthesia (ASRA) and the European and Scandinavian Societies of Anaesthesiology published guidelines for regional anaesthesia in 2010 for patients on anticoagulants. ${ }^{[1]}$ Various new oral anticoagulants have been approved by the US Food and Drug Administration (FDA) like dabigatran, rivaroxaban and ticagrelor and apixaban and are approved for the prevention of Venous thromboembolism (VTE) after elective knee or hip replacement surgery, for the prevention of stroke in patients with non-valvular $\mathrm{AF}$, and the treatment of VTE and prevention of recurrent VTE. ${ }^{[2]}$

Venous thromboembolism (VTE), deep vein thrombosis (DVT) and pulmonary embolism (PE), cause significant morbidity, mortality and healthcare costs. ${ }^{[3,4]}$ The risk of VTE is particularly high in patients who undergo major orthopedic surgery like total knee or hip replacement hence these patients should routinely receive anticoagulants for short-term perioperative and postoperative prophylaxis. ${ }^{[5,6]}$ Additionally, the use of regional neuraxial anesthesia poses a risk of hematoma in patients who are receiving anticoagulants, during insertion and removal of the needle and catheter.
In Europe, rivaroxaban is also indicated for co-administration with acetylsalicylic acid (ASA) alone or with ASA plus clopidogrel or ticlopidine for the prevention of atherothrombotic events after acute coronary syndrome with elevated cardiac biomarkers. The Study Group in France on thrombosis and haemostasis in surgery suggested adjustments to the interval between discontinuation of the drugs and performance of neuraxial procedures, based on the degree of risk of thrombosis. ${ }^{[7]}$

Inspite of the clinical evidence on the safety and efficacy profile of direct oral anticoagulants which has been demonstrated in clinical trials, anaesthesists need to be careful of how to minimize the risks of bleeding complications when treating patients who are taking an anticoagulant and are due to undergo surgery with regional anesthesia.

Rivaroxaban, a non-vitamin $\mathrm{K}$ antagonist direct oral anticoagulants (DOACs), was shown to be more effective than enoxaparin regimens for the prevention of VTE after orthopedic surgery. ${ }^{[8]}$

It has been shown that the timing of the first postoperative dose of thromboprophylaxis agent and the type of anesthesia used either general or neuraxia, during orthopaedic surgery may affect the clinical outcomes in patients undergoing surgery. ${ }^{[9]}$ Neuraxial anesthesia was shown to significantly reduce mortality and the occurrence of pulmorary embolism and deep vein thrombosis. ${ }^{[10]}$ 


\section{Aims and Objectives}

Study was carried out to look for the influence of timing of the first thrombo prophylactic dose on clinical outcomes in patients undergoing orthopedic surgery

\section{Patients and methods}

Patients aged $\geq 18$ years, with planned orthopaedic surgery or fracture-related orthopedic surgery and in whom thromboprophylaxis has been indicated. Written informed consent was taken from all the patients included in the study. The study protocol was approved by the Ethics Committee of the institute.

The type, dose, and duration of thromboprophylaxis were decided by the physician treating the patient. Total 324 patients were selected for the study for a period of 1 year at CCM Medical College and hospital Kachandur, Durg.

Out of 324 patients selected for the study 164 received rivaroxaban $10 \mathrm{mg}$ once daily and 160 received standard-of-care (SOC) pharmacological prophylaxis which includes low-molecular-weight heparins and dabigatran. Incidences of symptomatic thromboembolic events and bleeding events were analysed and recorded according to timing of the first postoperative thromboprophylactic dose and use of type of anaesthesia. Use of mechanical prophylaxis like elastic stockings and intermittent pneumatic compression was also recorded.
Bleeding events occurred during and after surgery were differentiated as major and non-major bleeding events. Major bleedings were clinically overt bleeding that was fatal occurring in critical organ which necessitated reoperation or was outside of the surgical site, and bleeding warranting treatment cessation and surgical-site bleeding events associated with a fall in hemoglobin of $\geq 2 \mathrm{~g} / \mathrm{dl}$. $^{[11]}$

\section{Analysis}

Incidences were calculated for the rivaroxaban and SOC groups. Bleeding events were those who presented as treatment-emergent events and thromboembolic events were those that occurred within 3 months of surgery.

Data was analysed according to the timing of the first postoperative dose of rivaroxaban and SOC and type of anesthesia used like general, spinal, combination, neuraxial, peripheral etc. mechanical prophylaxis used was also recorded

\section{Results}

All patients started antithrombotic therapy within 24 hours of surgery.

324 patients were selected for the study out of which 164 received rivaroxaban $10 \mathrm{mg}$ once daily and 160 received standard-of-care (SOC) pharmacological prophylaxis

Table 01

\begin{tabular}{|l|c|c|}
\hline Timing of first dose after surgery & Rivaroxaban $(\boldsymbol{\%})$ & Standard-of-care (SOC) (\%) \\
\hline$<\mathbf{6}$ hours & $74(45.12)$ & $70(43.75)$ \\
\hline$>\mathbf{6}$ to $\leq \mathbf{1 2}$ hours & $54(32.93)$ & $51(31.87)$ \\
\hline$>\mathbf{1 2}$ to $\leq \mathbf{2 4}$ hours & $36(21.95)$ & $39(24.38)$ \\
\hline Total & 164 & 160 \\
\hline
\end{tabular}

Out of 164 patients received rivaroxaban $74(45.12 \%)$ received first dose in $<6$ hours, $54(32.93 \%)$ received first dose between $>6$ to $\leq 12$ hours and $36(21.95 \%)$ received their first dose in $>12$ to $\leq$ 24 hours. In SOC group first dose given at $<6$ hours was 70
(43.75\%), patients who received first dose in $>6$ to $\leq 12$ hours was $51(31.87 \%)$ and $39(24.38 \%)$ received their first dose in $>12$ to $\leq$ 24 hours.

Table 02

\begin{tabular}{|l|c|c|c|c|c|}
\hline Adverse Events & Timing of first dose after surgery & Rivaroxaban & \% (n=164) & standard-of-care & \% (n=160) \\
\hline \multirow{4}{*}{$\begin{array}{l}\text { Major bleeding } \\
\text { events }\end{array}$} & $<6$ hours & 2 & 1.22 & 3 & 1.88 \\
\hline \multirow{3}{*}{$\begin{array}{l}\text { Thromboembolic } \\
\text { events }\end{array}$} & $>6$ to $\leq 12$ hours & 4 & 2.44 & 6 & 3.75 \\
\cline { 2 - 6 } & $>12$ to $\leq 24$ hours & 3 & 1.83 & 4 & 2.50 \\
\cline { 2 - 6 } & Total & 9 & 5.49 & 3 & 3 \\
\cline { 2 - 6 } & $>6$ hours & 2 & 13 & 4 & 2.13 \\
\cline { 2 - 6 } & $>12$ to $\leq 12$ hours $\leq 24$ hours & 4 & 2.44 & 4 & 2.50 \\
\hline
\end{tabular}

In $<6$ hours group, patients who received rivaroxaban major bleeding events was observed in $2(1.22 \%)$ cases. While in SOC group major bleeding events was observed in $3(1.88 \%)$. In $>6$ to $\leq$ 12 hours group, patients major bleeding events was observed in 4 (2.44\%) cases who received rivaroxaban, while in SOC group major bleeding events was observed in $6(3.75 \%)$. In $>12$ to $\leq 24$ hours groups major bleeding events for patients receiving rivaroxaban and SOC group was $3(1.83 \%)$ and $4(2.50 \%)$ respectively.
Thromboembolic events $<6$ hours group, patients who received rivaroxaban was $1(0.61)$ as compared to SOC group it was high 3 $(1.88 \%)$. Also in $>6$ to $\leq 12$ hours group patient who received rivaroxaban thromboembolic events was $2(1.22 \%)$ while in SOC group it was high $4(2.50 \%)$. But in $>12$ to $\leq 24$ hours group thromboembolic events was near about same in patients receiving rivaroxaban $4(2.44 \%$ ) and SOC group $4(2.50 \%)$. But the overall thromboembolic effect was lower in rivaroxaban as compared to SOC group. 


\section{International Journal of Innovative Research in Medical Science (IJIRMS) \\ Volume 03 Issue 06 June 2018, ISSN No. - 2455-8737 \\ Available online at - www.ijirms.in}

Overall major bleeding events observed in rivaroxaban group was $9(5.49 \%)$ was much lower than the SOC group which was 13 $(8.13 \%)$.

The percentage of patients using mechanical methods alongside pharmacological thromboprophylaxis was slightly higher in the rivaroxaban as compared to SOC groups (64\% and 55\%).

\section{Discussion}

Venous thromboembolism, comprising deep vein thrombosis cause significant morbidity, mortality in patients after orthopaedic surgery. The direct oral anticoagulants like apixaban, rivaroxaban, and dabigatran are approved for the prevention of VTE after orthopaedic surgery. Rivaroxaban which is direct inhibitors of Factor Xa has half-life of 5-13 hours. Elderly patients are at increased risk of thromboembolic disorders are more likely to be receiving anticoagulants. ${ }^{[12]}$

Earlier studies shown that timing of the first dose of thromboprophylaxis may have an impact on bleeding and thromboembolic events. Fondaparinux in a study was associated with the incidence of major bleeding events. ${ }^{[13]}$ While starting rivaroxaban 6-10 hours after surgery when hemostasis has been established is recommended. ${ }^{[14]}$

In our study major bleeding incidence was observed when rivaroxaban or SOC was given $>6$ to $\leq 12$ hours i.e. $4(2.44 \%)$ and $6(3.75 \%)$. In SOC group it was higher as compare to the rivaroxaban group. Overall major bleeding events were more in standard-of-care group $13(8.13 \%)$ as compared to rivaroxaban group which was $9(5.49 \%)$.

Thromboembolic events in patients receiving rivaroxaban was 7 $(4.27 \%)$ which was less than the SOC group 11 (6.88\%). In both the group overall thromboembolic effect in patients who received prophylaxis in $<6$ hours was less whiles in other group it was slightly higher.

These data suggest that in certain situations a slight delay in initiating rivaroxaban may not compromise the effectiveness of rivaroxaban in preventing thromboembolic events in these patients also use of mechanical thromboprophylaxis did not appear to further reduce the risk of thromboembolic events. ${ }^{[15]}$

Type of anaesthesia used may potentially influence clinical outcomes. Patients receiving anticoagulants and undergoing neuraxial anaesthesia may have an increased risk of spinal hematoma. ${ }^{[16]}$ In a study it was shown that rivaroxaban showed a favourable benefit-risk profile compared with SOC irrespective of the type of anaesthesia used. Some earlier studies have showed that neuraxial anaesthesia was associated with a lower incidence of VTE. $^{[17]}$

\section{Conclusion}

Comparison of rivaroxaban \& SOC shows the effectiveness and safety of rivaroxaban in patients undergoing major orthopaedic surgery in clinical practice. Major bleeding events \& thromboembolic with rivaroxaban were less as compared to SOC group. However owing to the small patient numbers in study group definitive conclusion cannot be drawn and further large studies are required for confirmations.

\section{References}

[1] Horlocker TT, Wedel DJ, Rowlingson JC, et al. Regional anesthesia in the patient receiving antithrombotic therapy or thrombolytic therapy: American Society of Regional Anesthesia and Pain Medicine evidence-based guidelines (third edition), Reg Anesth Pain Med, 2010, vol. 35(pg. 64-101)

[2] Cappelleri G., Fanelli A. Use of direct oral anticoagulants with regional anesthesia in orthopedic patients (2016) Journal of Clinical Anesthesia, 32 , pp. 224-235

[3] Deitelzweig, S.B., Johnson, B.H., Lin, J., and Schulman, K.L. Prevalence of clinical venous thromboembolism in the USA: current trends and future projections. Am J Hematol. 2011; 86: 217-220

[4] Spyropoulos, A. and Lin, J. Direct medical costs of venous thromboembolism and subsequent hospital readmission rates: an administrative claims analysis from 30 managed care organizations. $J$ Manag Care Pharm. 2007; 13: 475-486

[5] Falck-Ytter, Y., Francis, C.W., Johanson, N.A., Curley, C., Dhal, O.E., Schulman, S. et al. Prevention of VTE in orthopedic surgery patients: antithrombotic therapy and prevention of thrombosis, 9th ed: American College of Chest Physicians evidence-based clinical practice guidelines. Chest. 2012; 141: e278S-e325S

[6] Douketis, J.D., Spyropoulos, A.C., Spencer, F.A., Mayr, M., Jaffer, A.K., Eckman, M.H. et al.Perioperative management of antithrombotic therapy: antithrombotic therapy and prevention of thrombosis, 9th ed: American College of Chest Physicians evidence-based clinical practice guidelines. Chest. 2012; 141: e326S-e350S

[7] Sie P, Samama CM, Godier A, et al. Surgery and invasive procedures in patients on long-term treatment with direct oral anticoagulants: thrombin or factor-Xa inhibitors. Recommendations of the Working Group on Perioperative Hemostasis and the French Study Group on Thrombosis and Haemostasis, Arch Cardiovasc Dis , 2011, vol. 104 (pg. 669-76)

[8] Eriksson BI, Borris LC, Friedman RJ, Haas S, Huisman $M V$, Kakkar AK, Bandel TJ, Beckmann H, Muehlhofer E, Misselwitz F, Geerts W, RECORD1 Study Group. Rivaroxaban versus enoxaparin for thromboprophylaxis after hip arthroplasty. $N$ Engl J Med. 2008 Jun 26; 358(26):2765-75.

[9] Rosencher N, Bonnet MP, Sessler DI. Selected new antithrombotic agents and neuraxial anaesthesia for major orthopaedic surgery: management strategies. Anaesthesia. 2007 Nov; 62(11):1154-60.

[10] Rodgers A, Walker N, Schug S, McKee A, Kehlet H, van Zundert A, Sage D, Futter M, Saville G, Clark T, MacMahon $S$. $f$ Reduction of postoperative mortality and morbidity with epidural or spinal anaesthesia: results from overview of randomised trials. BMJ. 2000 Dec 16; 321(7275): 1493.

[11] European Medicines Agency Guideline on Clinical Investigation of Medicinal Products for Prevention of Venous Thromboembolism (VTE) in Patients Undergoing High VTE-Risk Surgery.2012.Availablefrom:http://www.ema.europa.eu/ 
docs/en_GB/document_library/Scientific_guideline/2012 /05/WC500127902.pdf.

[12] Landefeld, C.S. and Beyth, R.J. Anticoagulant-related bleeding: clinical epidemiology, prediction, and prevention. Am J Med. 1993; 95: 315-328

[13] Turpie AG, Bauer KA, Eriksson BI, Lassen MR. Fondaparinux vs enoxaparin for the prevention of venous thromboembolism in major orthopedic surgery: a metaanalysis of 4 randomized double-blind studies. Arch Intern Med. 2002 Sep 9; 162(16):1833-40.

[14] Bayer Pharma AG Xarelto® (Rivaroxaban) Summary of Product Characteristics. 2015. Availablefrom:http://www.ema.europa.eu/docs/en_GB/d ocument_library/EPAR_Product_Information/human/0 00944/WC500057108.pdf.

[15] Sylvia Haas, Gerlind Holberg, Reinhold Kreutz, Michael Rud Lassen, Lorenzo Mantovani, Verena Haupt, Kai Vogtländer, Alexander GG Turpie. The effects of timing of prophylaxis, type of anesthesia, and use of mechanical methods on outcome in major orthopedic surgery subgroup analyses from 17,701 patients in the XAMOS study. Vasc Health Risk Manag. 2016; 12: 209-218.

[16] Gogarten W, Vandermeulen E, Van Aken H, Kozek S, Llau JV, Samama CM, European Scoeity of Anaesthesiology. Regional anaesthesia and antithrombotic agents: recommendations of the European Society of Anaesthesiology. Eur J Anaesthesiol. 2010 Dec; 27(12):999-1015. 